\title{
Jungquartäre Taleintiefung und Flächenbildung am Wilseder Berg (Lüneburger Heide)
}

\author{
Von Karsten GarlefF, Göttingen und Sotirios N. Leontaris, Athen
}

Mit 3 Abbildungen

\begin{abstract}
$\mathrm{Zus}$ a m m e $\mathrm{n}$ a s s u $\mathrm{ng}$. Geomorphologische Untersuchungen in der Lüneburger Heide, einer Altmoränenlandschaft im Bereich warthezeitlicher Eisrandlagen in NW-Deutschland, ergaben Hinweise auf Art und Ausmaß der überwiegend periglazialen Umformung des glazigenen Reliefs. Effekte der Umformung sind in Abhängigkeit vom Ausgangsrelief einerseits Talbildung, andererseits Einebnung. Die Taleintiefung bewirkte stellenweise Reliefverstärkung, die Einebnung dagegen Reliefverringerung um $10-15 \mathrm{~m}$. Eine Einebnungsfläche wurde anhand eeminterglazialen Torfes und eines fossilen Bodens sowie mineralischer Sedimente als weichselzeitlich periglaziale Fußfläche datiert. Ihre Entstehung verdankt sie vorwiegend flächenhaften Abflußvorgängen, bei denen Hohlformen aufgefüllt und Kuppen eingeebnet wurden. Die Untersuchung und Kenntnis der periglazialen Umformung kann wichtige Beiträge zur Klärung der Morphogenese der Altmoränengebiete leisten.

$\mathrm{R}$ és u mé. Au cours des investigations géomorphologiques dans la Lüneburger Heide, une région morainique quaternaire datée de l'époque Warthe en Allemagne Nord-Ouest, on a trouvé des indications aux tendences et aux quantités de la déformation du relief glaciaire. Cette déformation se passe surtout dans un milieu périglaciaire. Les effets les plus importants de la déformation sont d'une part l'élargissement des vallées sur le relief fort, d'autre part un nivellement du relief faible. Cet approfondissement des vallées effectuait un élargissement de l'altitude relative sur certaines parties de 10 à $15 \mathrm{~m}$. Le nivellement du relief faible effectuait au contraire une diminution du relief de 10 à $15 \mathrm{~m}$. On a prouvé le développement d'une plaine comme glacis périglaciaire par des tourbes éemiennes, des sols fossils et des sédiments minéraux. Pour son développement les processus du ruisselement étaient d'une grande importance; à la fois par ablation et par dépôt. La connaissance de la déformation périglaciaire peut prendre une place préférée à l'investigation de la morphogenèse des régions morainiques quaternaires.
\end{abstract}

\section{Problemstellung und Untersuchungsgebiet}

Forschungen zur Morphogenese treffen in Altmoränengebieten auf Schwierigkeiten, die z. T. durch das schwer erfaßbare Ausmaß der Umgestaltung des glazigenen Reliefs bedingt sind. Diese Umformung, bei der die Reliefenergie verringert, Täler - insbesondere die heutigen Trockentäler - und äolische Kleinformen gebildet sowie geschlossene Hohlformen vernichtet wurden (GRIPP 1924), betrifft zunächst den Kleinformenschatz. Um Hinweise auf die Prozesse der Umformung sowie auf das Ausgangsrelief zu erlangen, wurde der Kleinformenschatz im weiteren Bereich um den Wilseder Berg, einem Ausschnitt aus der nordwestdeutschen Altmoränenlandschaft, untersucht. Das Gebiet gehört zu einem der $\mathrm{N}-\mathrm{S}$-streichenden Höhenzüge der nordwestlichen Lüneburger Heide. Der südliche Teil des Höhenzuges, das engere Untersuchungsgebiet (Abb. 1 u. Top. Karte 1:25000 Nr. 2825), wird durch kleinere Rücken und Kuppenreihen mit westöstlichen Richtungen modifiziert. Von den Hochgebieten in $120-170 \mathrm{~m}$ NN leiten meist flache Abdachungen zu Flächen in 60-90 m NN über. Am Fuß steilerer Hänge sind Fußflächen eingeschaltet, wie z. B. nördlich des Wilseder Berges. Die Abdachungen sind in Abhängigkeit vom Böschungsgrad unterschiedlich dicht zertalt. Die Täler sind überwiegend Trockentäler. Perennierende Wasserführung ist nur in wenigen größeren Tälern unterhalb $90-115 \mathrm{~m} \mathrm{NN}$ im Bereich der Bachkerben und Talauen zu beobachten. Außer den genannten sind äolische Kleinformen weit verbreitet. Der Effekt der äolischen Formung ist allerdings so gering, daß er in diesem Zusammenhang nicht näher beschrieben zu werden braucht.

Die größeren Formen werden im diesbezüglichen Schriftum als Bildungen im Bereich warthezeitlicher Eisrandlagen gedeutet (Woldstedt 1955). Der Verlauf der Eisrandlagen 
im einzelnen, ihre stratigraphische Stellung und der Anteil älterer Formen sind jedoch noch umstritten (LÜTTIG 1968).

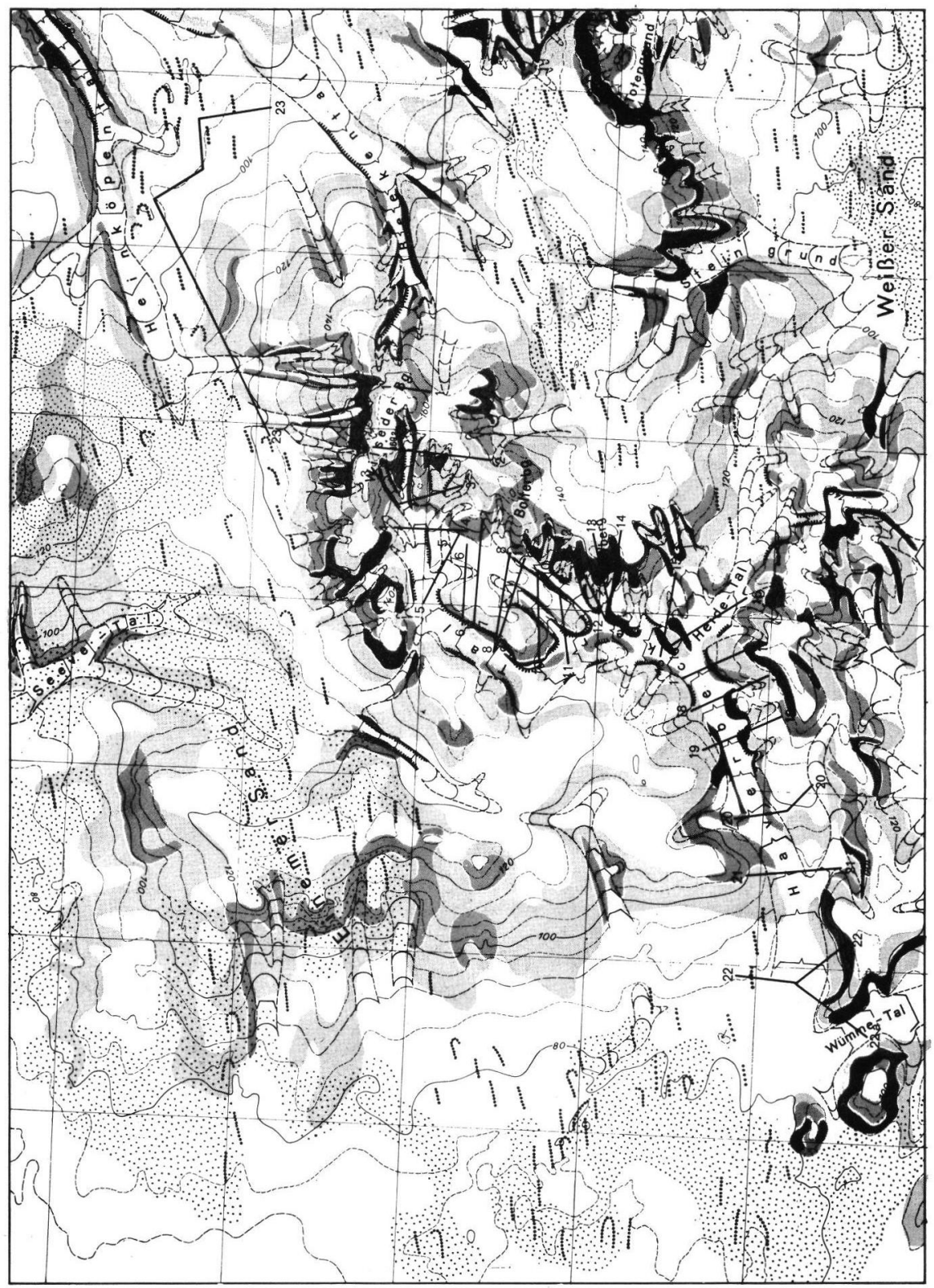

Abb. 1. Morphographische Karte des Untersuchungsgebietes. (Die Profillinien 1-23 zwischen Wümmetal und Heinköppental sind randparallel um $7 \mathrm{~mm}$ nach rechts verschoben.)

\section{Das Haverbecke-Tal}

Ein Beispiel für die Zertalung ist das Haverbecke-Tal, das vom Wilseder Berg S-förmig nach W und S zieht und westlich Niederhaverbeck auf der Fläche des Wintermoorer Sanders ausläuft. Das Talsystem der Haverbecke weist in Grundriß, Dichte und Ausbildung 
auf Abhängigkeiten von vorgegebenen Reliefverhältnissen hin: Die stärker geneigten Abdachungen sind von zahlreichen meist gestreckten und stark verästelten Kerb- und Sohlentälchen zerfurcht, deren Querprofil meist regelhaften Wandel von flachen Einzugsmulden über Kerbtalstrecken zu Sohlentälern zeigt. Zwischen Sohle und Hang sind häufig Übergangsböschungen eingeschaltet, die durch humoses Schwemm-Material gebildet werden. Im Bereich geringer Reliefenergie treten dagegen ausschließlich flache Muldentälchen auf. Weite Verbreitung haben asymmetrische Talstrecken. Nach Ausbildung und Verteilung entspricht diese Talasymmetrie den klimatisch bedingten primären und sekundären Asymmetrien POSER's (1947).

Formen und Verteilung der Tälchen des Haverbecke-Talsystems entsprechen den Verhältnissen „reifzertalter Altmoränenlandschaften“, wie sie z. B. von GRIPP (1924), J.HAGEDORN (1964) u. a. beschrieben wurden. Sie gleichen damit in vielen Merkmalen den Tälern rezenter Periglazialgebiete (Poser 1936). Dementsprechend können die Täler als vorzeitliche Bildungen unter periglazialen Bedingungen gedeutet werden. In diese Vorzeitformen sind Bachkerben, Talauen und steilhängige trockene Erosionsrisse deutlich als jüngere Bildungen eingeschnitten.

An den Talhängen treten häufig sowohl einfache Hangknicke als auch deutlich ausgebildete Terrassen auf (Abb. 1 u. 2, Profile 1-22). Sie können zwanglos miteinander verknüpft werden und belegen 5 Stadien der Talentwicklung ( $\mathrm{a}-\mathrm{e}$ in Abb. 2). Die unteren Stadien sind durch ihre Beziehungen zu Akkumulationsmassen gekennzeichnet. So ist die

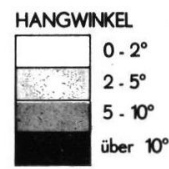

0

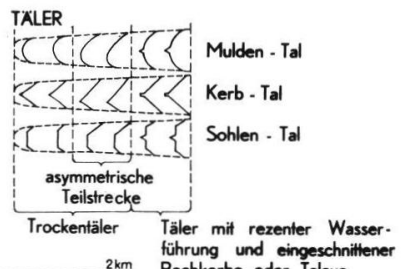

$2 \mathrm{~km}$ Bührung und eingeschniller

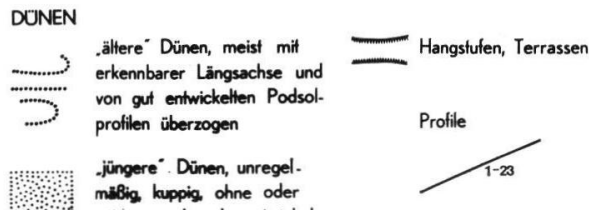

misigg kuppig. ohne oder

mit nur schwach

Talaue (e) in weiten Teilen eine holozäne Aufschüttungssohle, die auf bis zu 3-4 m mächtigen sandig-humosen Sedimenten mit Torfeinschaltungen liegt. Diese holozänen Sedimente sind zu einem geringen Teil durch anthropogene Maßnahmen entstanden, wie besonders an den Rändern der Talaue zu erkennen ist. Ein nicht genauer abschätzbarer Anteil dürfte durch anthropogene Veränderungen der Abtragungsbedingungen im Einzugsgebiet in das Tal und zur Ablagerung gekommen sein. Über den Beginn der Sedimentation liegen keine Anhaltspunkte vor, da Datierungen von Sedimenten aus den untersten Partien der Talfüllung noch ausstehen.

Die holozäne Talfüllung liegt in einem Tal (d), das in die älteren, weithin von einer windkanterführenden Steinsohle überzogenen Terrassen und Hänge eingeschnitten ist. Die Steinsohle ist nach eigenen Beobachtungen und zahlreichen Untersuchungen (J. HAGEDORN 1964) eine hoch- bis spätglaziale Oberflächenbildung. Demnach muß die Zerschneidung des Talstadiums c spätglazial oder später erfolgt sein. Die Parallelisierung dieser Einschneidung mit der spätglazialen Erosionsphase in den großen Tälern des nordwestdeutschen Tieflandes liegt nahe. Rückwirkungen der glazialeustatischen Meeresspiegelschwankungen, wie sie in diesen Tälern nachgewiesen wurden (Woldstedt 1955), können dagegen aufgrund des Konvergierens der Talböden und Terrassen auf der Fläche des Wintermoorer Sanders als der lokalen Erosionsbasis für diese und frühere Phasen ausgeschlossen werden. Demnach müssen sowohl die Erosionsphasen als auch die Akkumulationsphase hier im $\mathrm{Ge}-$ gensatz zu der von RoHDEnburg (1968) vertretenen Auffassung auf klimatisch und evtl. teilweise anthropogen gesteuerten Veränderungen der Abtragungs- und Transportverhältnisse beruhen. 
Von den älteren Talstadien ist das der Trockentalböden bzw. unteren Terrasse (c) durch Vergleich mit den Verhältnissen benachbarter Gebiete (J. HAgedorn 1964), durch die Verknüpfung mit der Steinsohle und durch Beobachtungen auf der Fußfläche nördlich des Wilseder Berges als weichselzeitlich zu datieren.
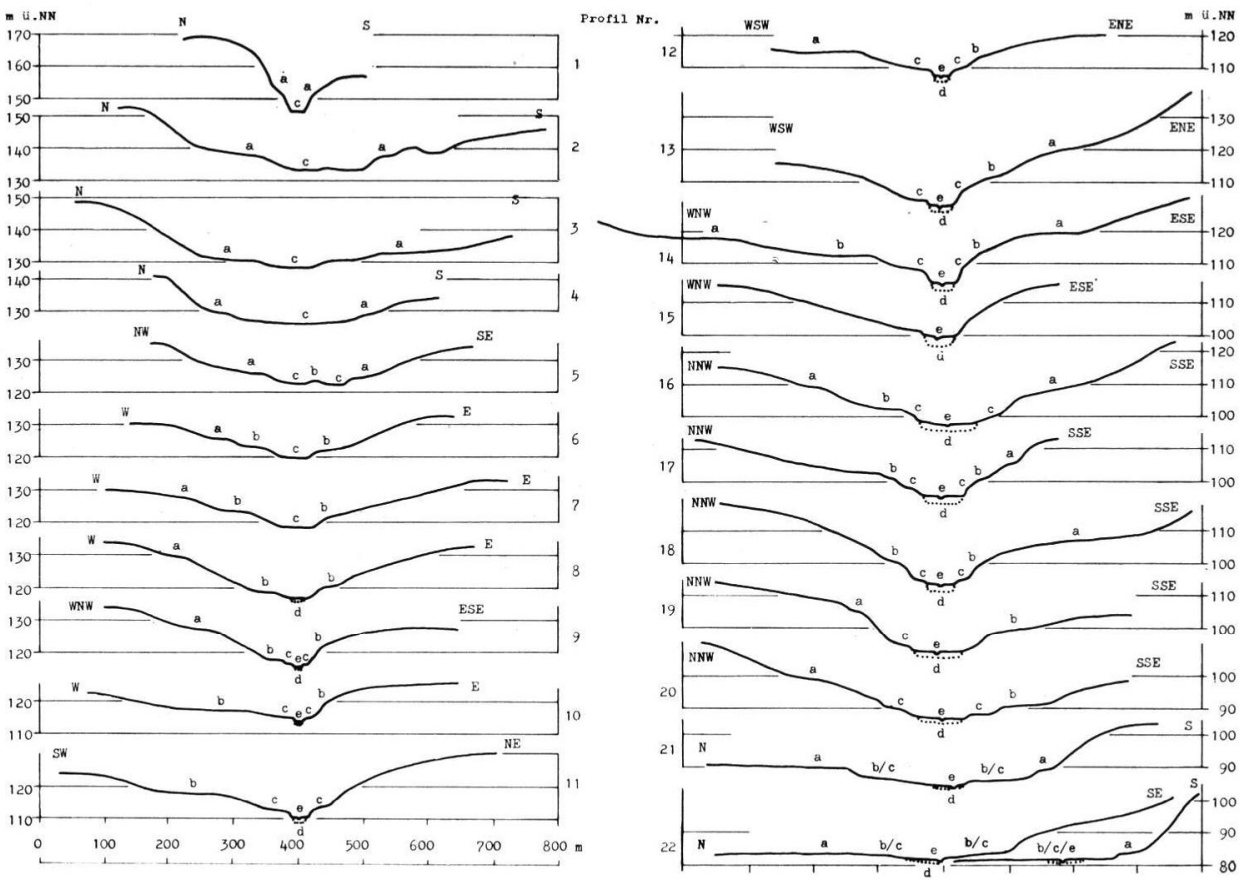

Abb. 2. Profile durch das Haverbecke-Tal, 5 fach überhöht.

Die Reste des Talstadiums a sind mit einer Fußfläche südlich des Wilseder Berges, d. h. am distalen Rand warthezeitlicher Endmoränen, verknüpft. Danach könnte dieses Stadium eine warthezeitliche glazifluviale Abflußbahn gewesen sein, die in Schwemmfächern vor dem Eisrand wurzelnd über einen kleinen Rinnensander zum Wintermoorer Sander führte. Diese Datierung wird gestützt durch Beobachtungen im Heide-Tal. Hier liegt eine beckenartige Erweiterung des Talgrundes einige Meter tiefer als die Reste des Stadiums a im anschließenden Teil des Haverbecke-Tales. Die Erweiterung ist über einer stellenweise mehr als $10 \mathrm{~m}$ mächtigen Folge von Beckensedimenten, schluffigen Feinsanden mit dunklen Lehmbändern, ausgebildet, die vermutlich in einem glazigenen Becken zwischen Stadtberg und Oberhaverbecker Rücken abgelagert wurde. Die relative Höhenlage der Beckensedimente zum Talstadium a im Haverbecke-Tal deutet darauf hin, daß z. Z. des vermuteten glazifluvialen Abflusses das Becken noch nicht vorhanden, bzw. noch durch Toteis ausgefüllt war. Das Becken kann nach Höhenlage und Verknüpfungsmöglichkeit mit den Resten des Talstadiums b erst während und nach dessen Entstehung aufgefüllt worden sein. Daraus ergibt sich für das Talstadium b ein spätwarthezeitliches Alter.

Die Befunde zeigen, daß auch in kleinen Talsystemen im Bereich warthezeitlicher Moränen ein mehrphasiger Entwicklungsgang zu erkennen ist. Die Taleintiefung erfolgte phasenhaft und ist aus den Resten der Erosionssohlen und der zugehörigen Hänge zu rekonstruieren. Sie bewirkte im mittleren Teil des Talsystems Reliefverstärkung um 10-15 m. Die jüngste, holozäne Formung führte dagegen streckenweise zu geringer Aufhöhung des 
Talbodens. Die Formungsphasen sind vorwiegend durch klimatisch gesteuerte Veränderungen der Abtragungs- und Transportbedingungen verursacht. Dabei wurden in Abhängigkeit von Belastung und Abflußregime verschiedene Längsgefällskurven ausgebildet, die sich in der unterschiedlichen Höhendifferenz der Talbodenreste im Verlauf des Tales zu erkennen geben.
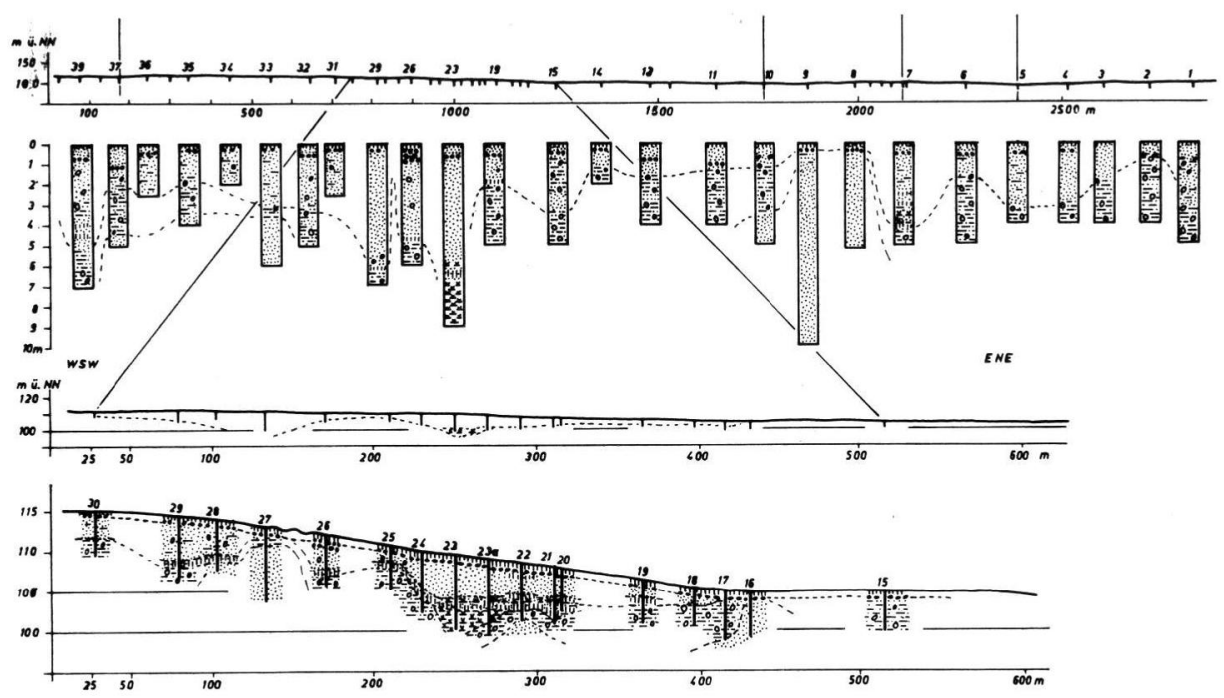

Profil 23 (unteres Profil 5fach uberhöht)

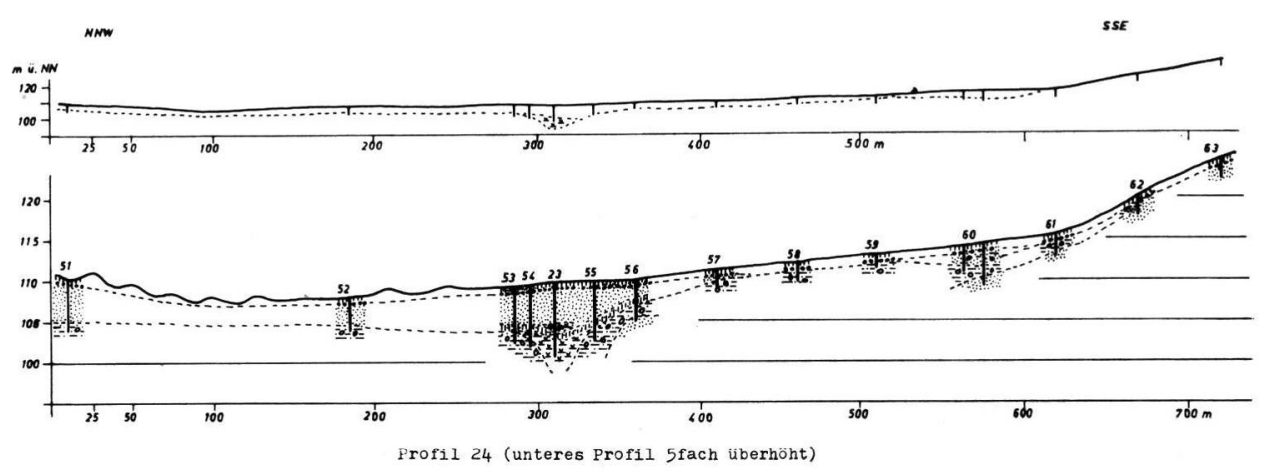

$\begin{array}{ll}\text { Sand } & \\ \text { Steinsohle } \\ \text { Lehm } & \square \text { Materialgrenze } \\ \text { Steine } & \text { Bohrung } \\ \text { Knick im Profil }\end{array}$

Abb. 3. Fußfläche nördlich des Wilseder Berges. 


\section{Die Flußfläche nördlich des Wilseder Berges}

Der N-Hang des Wilseder Berges fällt mit Hangneigungen um und über $10^{\circ} 20-50 \mathrm{~m}$ zu einer stellenweise dünenüberlagerten Fußfläche ab. Er wird von Trockentälern gegliedert, die nach wenigen Hundert Metern Laufstrecke auf der Fußfläche enden. Diese gewinnt im NW Anschluß an das obere Seeve-Tal, im NE und E an Seitentäler des AueRadenbach-Talsystems.

Die Fußfläche (Abb. 1 u. 3, Profile 23 u. 24) ist weithin von 0,2-0,6 m mächtigem Flugsand bedeckt, der einer windkanterführenden Steinsohle aufliegt und im Bereich der Dünen stellenweise mehr als $5 \mathrm{~m}$ Mächtigkeit erreicht. Gelegentlich enthält der Flugsand überwehte Böden oder deren Reste. Die Steinsohle im Liegenden des Flugsandes bedeckt meist einen unterschiedlich sandigen Geschiebelehm oder eine lehmige Solifluktionsdecke. Diese Schicht wurde in wechselnder Tiefe und Mächtigkeit in weiten Teilen der Fußfläche festgestellt. Sie liegt auf Mittel- und Grobsanden. Der Geschiebelehm ist nach geschiebestatistischen Untersuchungen LüTTIG's (frdl. mdl. Mitt.) warthezeitlich. Er keilt am Hang des Wilseder Berges sowie an zahlreichen Stellen der Fußfläche aus. Hier wird er vertreten durch steinführenden Sand oder lediglich durch die Steinsohle. In der Umgebung derartiger Stellen konnten vereinzelt Steinanreicherungen bis über $1 \mathrm{~m}$ Mächtigkeit festgestellt werden, die offenbar durch Umlagerung und selektive Abtragung aus dem Geschiebelehm hervorgegangen sind. Stellenweise taucht die Geschiebelehmoberfläche ab und wird von schluffigen Fein- und Mittelsanden überlagert, die in ihren unteren Partien häufig umgelagerte Bodenreste enthalten. Im Bereich ihrer Tieflagen trägt die Geschiebelehmoberfläche meist einen Boden oder dessen Reste, gelegentlich auch eine geringmächtige Torfschicht. In einer etwa $30 \times 50 \mathrm{~m}$ weiten Hohlform in der Geschiebelehmoberfläche wurde über $2,5 \mathrm{~m}$ mächtiger Torf erbohrt, der von verschwemmtem Bodenmaterial, einem Torfband sowie etwa $6 \mathrm{~m}$ Schwemmsanden unter Steinsohle und Flugsandhaut überlagert war. Der Pollengehalt des Torfes repräsentiert den Ausgang einer Warmzeit von der Eichenmischwaldphase bis zum Vorherrschen der Nichtbaumpollen. Taxus- und insbesondere reichlich Carpinus-Pollen sowie der Mangel an Fagus-Pollen kennzeichnen den Torf als eemzeitlich. Er ist randlich mit dem Boden an der Oberfläche des Geschiebelehms verknüpft, so daß dieser ebenfalls als eemzeitlich zu datieren ist.

Die Schichtenfolge im Bereich der Fußfläche gestattet die Rekonstruktion der Morphogenese. Danach handelte es sich zunächst um ein warthezeitliches Grundmoränengebiet mit Kuppen und Söllen. Während der Eemwarmzeit entwickelte sich an der Oberfläche ein Boden, in den Hohlformen wurde organogenes Sediment abgesetzt. In der Weichselkaltzeit wurde Material von den Hängen und Kuppen abgetragen und z. T. in den Senken abgelagert. Dabei war nach Ausweis der korrelaten Sedimente vorwiegend flächenhafte Verschwemmung wirksam. Das im Bereich der Fußfläche sedimentierte Material wurde teilweise durch die Tälchen herantransportiert. Dieser Transport hat sich am Fuß der steileren Abdachung offenbar flächenhaft ausgeweitet. Dabei wurden kleinere Kuppen unterschnitten und bis ins Niveau der Schwemmebene abgetragen. Erst nach teilweisem Verlust der Schuttlast trat erneut linienhafter Abfluß und Abtragung ein, entsprechend den Vorgängen, die Dege (1938) in rezenten, Lehmann (1948) in vorzeitlichen Periglazialgebieten erschlossen. Nach Ausbildung der Schwemmebene wurde durch Solifluktion in Verbindung mit flächenhafter aquatischer und äolischer Formung ein steinführender Schutt bzw. die Steinsohle abgelagert. Schließlich wurde diese Schicht von Flugsand überdeckt, der stellenweise während des Holozäns nochmals umgelagert wurde.

Die Befunde lassen wesentliche Veränderungen des Reliefs im Bereich der N-Abdachung des Wilseder Berges unter periglazialen Bedingungen erkennen. Auf den höheren Geländeteilen herrschte Abtragung, in den tiefgelegenen Sedimentation, die zur Ausbil- 
dung einer Schwemmebene führte, wobei kleinere Kuppen abgetragen und in die Fläche einbezogen wurden. Aus der Abschätzung des abgelagerten Materials einerseits, des Einzugsareals andererseits ergeben sich Anhaltspunkte für die Beurteilung der Reliefverringerung. Dabei wurde berücksichtigt, daß nur ein Teil des abgetragenen Materials im Bereich der Fußfläche abgelagert wurde. Danach sind die Senken stellenweise um mehr als $5 \mathrm{~m}$ aufgehöht, die Höhen um durchschnittlich 5-10 m erniedrigt worden. Die Verringerung der Höhenspanne zwischen dem Wilseder Berg und seinem nördlichen Vorland beträgt demnach seit der Eemwarmzeit 10-15 m. Ähnliche Werte ermittelten GRIPP (1939) und J. HAgedorn (1965) in anderen Bereichen des Altmoränengebietes.

\section{Tendenzen periglazialer Reliefentwicklung in Lockermaterial und ihre Bedeutung für die geomorphologische Forschung in Altmoränengebieten}

Die Beispiele lassen unterschiedliche Tendenzen der Umformung des glazigenen Ausgangsreliefs erkennen. Diese überwiegend periglaziale Umformung bewirkte einerseits Talbildung im Bereich der Abdachungen und Hauptabflußbahnen, andererseits Einebnung in Bereichen primär flacheren Reliefs. Der Ausgleich der Höhenunterschiede des glazigenen Reliefs scheint angesichts der ausgedehnten Flachrelief-Bereiche in Altmoränengebieten weit verbreitet gewesen zu sein. Durch die periglaziale Einebnung entstanden Flächen, deren Gefälle unter den Grenzwerten der Solifluktion liegt (Büdel 1937). Diese weitgehende Einebnung wurde im wesentlichen durch flächenhafte Abspülung bewirkt. Flächenhafte Abflußvorgänge waren offenbar am Fuße steilerer Abdachungen begünstigt und schufen hier Fußflächen unter periglazialen Bedingungen. Derartige pleistozäne Fußflächenbildung oder zumindest -erhaltung wurde bereits mehrfach aus Bereichen pleistozänen Lockermaterials und gering resistenter älterer Gesteine beschrieben (Mensching 1960, Rotnicki 1964, Czudek \& Demek 1970), doch konnte sie bisher nur selten exakt datiert werden. Durch den Nachweis jungpleistozäner Fußflächenbildung werden Hinweise auf Formungskonvergenzen zwischen periglazialen und semiariden bis ariden Bedingungen (MORTENSEN 1930, DYLIK 1957) gestützt.

Die Umformung des glazigenen Reliefs verlief im Bereich des untersuchten Altmoränengebietes in verschiedenen Phasen mit wechselnder Dom:nanz einzelner Prozesse. Ihre Differenzierung und Aufeinanderfolge ist allerdings hier noch nicht so weit zu erschließen, wie in anderen Bereichen (Dylik 1961, Semmel 1968). So konnte lediglich der Wechsel kalt- und warmzeitlicher Formung in den Tälern und die Abfolge des Vorherrschens aquatischer, solifluidaler und äolischer Formung auf der Fläche nachgewiesen werden.

Die Untersuchung der Kleinformen im Bereich der Altmoränen gibt sowohl Hinweise auf die Vorgänge und Tendenzen der überwiegend periglazialen Umformung als auch Anhaltspunkte für die Rekonstruktion des glazigenen Ausgangsreliefs. Die Analyse der Täler ist besonders aufschlußreich. Sie führt zu einer Differenzierung in - meist kleine Täler, die in gestrecktem Verlauf vorgegebenen Abdachungen folgen, und größere Täler, die unter Benutzung der Rinnen und Senken des Ausgangsreliefs in unregelmäßigen Windungen verlaufen. Diese Differenzierung und die Untersuchung der Täler hinsichtlich älterer Stadien ihrer Entwicklung lassen Schlüsse auf glazigene oder glazifluviale Formen sowie ihre z. T. kleinräumigen Altersdifferenzen zu, wie am Beispiel des Haverbeckeund Heide-Tales gezeigt wurde.

Untersuchung und Kenntnis der flächenhaften periglazialen Formung, insbesondere der flächenhaften Abtragung der Hochlagen, können zur Deutung und Datierung der Großformen beitragen. Bei Berücksichtigung der flächenhaften Erniedrigung der Höhen um $5-10 \mathrm{~m}$ und der mittleren Mächtigkeit der warthezeitlichen Grundmoräne in diesem Gebiet von $2-3 \mathrm{~m}$ ist damit zu rechnen, daß im Bereich der Hochlagen flächenhaft das 
Liegende dieser Grundmoräne zutage ansteht. Dementsprechend können die Befunde Hesemann's (1939), LütTig's (1958) und K. RichteR's (1959), nach denen als warthezeitliche Endmoränen angesehene Höhenzüge des Untersuchungsgebietes drenthezeitliches Geschiebeinventar aufweisen, dahingehend interpretiert werden, daß diese Höhen präwarthezeitlich angelegte Sockel enthalten, die warthezeitlich nur oberflächlich überformt wurden.

\section{Schriften}

BüDEL, J.: Eiszeitliche und rezente Verwitterung und Abtragung im ehemals nicht vereisten Teil Mitteleuropas. - Petermanns geogr. Mitt. Ergh., 229, 5-71, Gotha 1937.

Czudek, T. \& Demek, J.: Pleistocene Cryopedimentation in Czechoslovakia. - Acta geogr. Univ. lodz., 24, 101-108, Lódź 1970.

DEGE, W.: Geomorphologische Forschungen im nördlichen Andréeland. - Diss. Münster 1938.

DyLIK, J.: Tentative comparision of planation surfaces occuring under warm and under cold semiarid climatic conditions. - Biul. peryglac., 5, 175-186, Lódź 1957.

- : Analyse sédimentologique des formations de versant remplissant les dépressions fermées aux environs de Lódź. - Biul. peryglac., 10, 57-74, Lódź 1961.

GripP, K.: Uber die äußerste Grenze der letzten Vereisung in Nordwestdeutschland. - Mitt. geogr. Ges. Hamburg, 36, 159-245, Hamburg 1924.

- : Der Oberflächenabtrag im Alt-Diluvium und seine Bedeutung für das Vorkommen paläolithischer Funde. - Offa, 4, 59-82, Neumünster 1939.

Hagedorn, J.: Geomorphologie des Uelzener Beckens. - Göttinger geogr. Abh., 31, Göttingen 1964.

- : Die Umgestaltung des glazigenen Reliefs der norddeutschen Altmoränengebiete am Beispiel des Uelzener Beckens. - Eiszeitalter u. Gegenwart, 16, 116-120, Öhringen 1965.

Hesemann, J.: Diluvialstratigraphische Geschiebeuntersuchungen zwischen Elbe und Rhein. Abh. naturw. Ver. Bremen, 31, 247-319, Bremen 1939.

Lenmann, H.: Periglaziale Züge im Formenschatz der Veluwe. - Erdkunde, 2, 69-72, Bonn 1948.

Lüttig, G.: Methodische Fragen der Geschiebeforschung. - Geol. Jb., 75, 361-418, Hannover 1958.

- : Möglichkeiten der Endmoränen-Verknüpfung im Gebiet zwischen Aller und Elbe. - Mitt. geol. Institut Techn. Univ. Hannover, 8, 66-72, Hannover 1968.

Mensching, H.: Periglazial-Morphologie und quartäre Entwicklungsgeschichte der Hohen Rhön und ihres östlichen Vorlandes. - Würzburger geogr. Arb., 7, Würzburg 1960.

Mortensen, H.: Einige Oberflächenformen in Chile und auf Spitzbergen. - Petermanns geogr. Mitt. Ergh., 209, 147-156, Gotha 1930.

Poser, H.: Talstudien aus Westspitzbergen und Ostgrönland. - Z. Gletscherk., 24, 43-98, Berlin 1936.

— : Dauerfrostbaden und Temperaturverhältnisse während der Würmeiszeit im nicht vereisten Mittel- und Westeuropa. - Naturwissenschaften, 34, 10-18, Berlin 1947.

RichteR, K.: Geschiebegrenzen und Eisrandlagen in Niedersachsen. - Geol. Jb., 76, 223-234, Hannover 1959.

Rohdenburg, H.: Zur Deutung der quartären Taleintiefung in Mitteleuropa. - Die Erde, 99, 297-304, Berlin 1968.

RotNICKI, K.: Periglacial pediments and equiplanation surfaces in the Ostrzeszow Hills (push end moraine of the Warta stage). - Rep. VI. th. Internat. Congr. Quaternary, Warsaw 1961, IV, 139-142, Lódź 1964.

Semmel, A.: Studien über den Verlauf jungpleistozäner Formung in Hessen. - Frankfurter Geogr. Hefte, 45, Frankfurt 1968.

Woldstedt, P.: Norddeutschland und angrenzende Gebiete im Eiszeitalter. - 2. Aufl., Stuttgart 1955.

Manuskr. eingeg.: 9. 8. 1971.

Anschriften der Verf.: Dr. Karsten Garleff, Geogr. Institut, 34 Göttingen, Herzberger Landstr. 2; Dr. Sotirios N. Leontaris, Geomorphologisch-Tektonisches Institut der Universität Athen, Hippokratousstr. 33. 